\title{
$\exists+$ Sorafenib and everolimus for patients with unresectable high-grade osteosarcoma progressing after standard treatment: a non-randomised phase 2 clinical trial
}

\author{
Giovanni Grignani, Emanuela Palmerini, Virginia Ferraresi, Lorenzo D’Ambrosio, Rossella Bertulli, Sebastian Dorin Asaftei, Angela Tamburini, \\ Ymera Pignochino, Dario Sangiolo, Emanuela Marchesi, Federica Capozzi, Roberto Biagini, Marco Gambarotti, Franca Fagioli, \\ Paolo Giovanni Casali, Piero Picci, Stefano Ferrari, Massimo Aglietta, for the Italian Sarcoma Group
}

\section{Summary}

Lancet Oncol 2015; 16: 98-107 Published Online December 11, 2014 http://dx.doi.org/10.1016/ S1470-2045(14)71136-2

See Comment page 12

Medical Oncology, Candiolo Cancer Institute-FPO, IRCCS, Candiolo, Italy (G Grignani MD, LD'Ambrosio MD, Y Pignochino PhD, D Sangiolo PhD, F Capozzi BSC Prof M Aglietta MD); Chemotherapy, Musculoskeletal Oncology, IRCCS Istituto Ortopedico Rizzoli, Bologna, Italy (E Palmerini MD, E Marchesi PhD, S Ferrari MD); Medical Oncology A, IRCCS Regina Elena National Cancer Institute, Rome, Italy (V Ferraresi MD); Department of Oncology, University of Torino, Turin, Italy (LD'Ambrosio, Y Pignochino, D Sangiolo, F Capozzi, Prof M Aglietta);Adult Mesenchymal Tumor Medical Oncology Unit, Fondazione IRCCS Istituto Nazionale Tumori,

Milan, Italy (R Bertulli MD,

P G Casali MD); Pediatric OncoHematology, Città della Salute $e$ della Scienza di Torino, Turin, Italy (SD Asaftei MD,

F Fagioli MD); Paediatric

Onco-Hematology, Azienda Ospedaliero Universitaria Meyer Children Hospital, Firenze, Italy

(A Tamburini MD); Oncological Orthopaedics Department, IRCCS Regina Elena National Cancer Institute, Rome, Italy (R Biagini MD); Department of Pathology, IRCCS Istituto Ortopedico Rizzoli, Bologna, Italy (M Gambarotti MD); and Laboratory of Experimental Oncology, IRCCS Istituto Ortopedico Rizzoli, Bologna, Italy (P Picci MD)

Correspondence to:

Dr Giovanni Grignani, Medical Oncology, Candiolo Cancer Institute-FPO, IRCCS, 10060 Candiolo, Italy giovanni.grignani@ircc.it

Background Results of previous study showed promising but short-lived activity of sorafenib in the treatment of patients with unresectable advanced and metastatic osteosarcoma. This treatment failure has been attributed to the mTOR pathway and might therefore be overcome with the addition of mTOR inhibitors. We aimed to investigate the activity of sorafenib in combination with everolimus in patients with inoperable high-grade osteosarcoma progressing after standard treatment.

Methods We did this non-randomised phase 2 trial in three Italian Sarcoma Group centres. We enrolled adults ( $\geq 18$ years) with relapsed or unresectable osteosarcoma progressing after standard treatment (methotrexate, cisplatin, and doxorubicin, with or without ifosfamide). Patients received $800 \mathrm{mg}$ sorafenib plus $5 \mathrm{mg}$ everolimus once a day until disease progression or unacceptable toxic effects. The primary endpoint was 6 month progression-free survival (PFS). All analyses were intention-to-treat. This trial is registered with ClinicalTrials.gov, number NCT01804374.

Findings We enrolled 38 patients between June 16, 2011, and June 4, 2013. 17 (45\%; 95\% CI 28-61) of 38 patients were progression free at 6 months. Toxic effects led to dose reductions, or short interruptions, or both in 25 (66\%) of 38 patients and permanent discontinuation for two (5\%) patients. The most common grade 3-4 adverse events were lymphopenia and hypophosphataemia each in six (16\%) patients, hand and foot syndrome in five (13\%), thrombocytopenia in four (11\%), and fatigue, oral mucositis, diarrhoea, and anaemia each in two (5\%). One patient (3\%) had a grade 3 pneumothorax that required trans-thoracic drainage, and that recurred at the time of disease progression. This was reported as a serious adverse event related to the study drugs in both instances. No other serious adverse events were reported during the trial. There were no treatment-related deaths.

Interpretation Although the combination of sorafenib and everolimus showed activity as a further-line treatment for patients with advanced or unresectable osteosarcoma, it did not attain the prespecified target of 6 month PFS of $50 \%$ or greater.

Funding Italian Sarcoma Group.

\section{Introduction}

High-grade osteosarcoma is a rare sarcoma affecting roughly 1135 new patients every year in the European Union. ${ }^{1}$ A multidisciplinary treatment including chemotherapy and complete surgical excision of the tumour cures roughly $70 \%$ of patients. ${ }^{2}$ The best predictor of cure remains complete tumour resection with adequate margins both in the localised ${ }^{3}$ and relapsed or metastatic settings. ${ }^{4,5}$ Unfortunately, although the most active chemotherapy of high-dose methotrexate, doxorubicin, and cisplatin, with or without ifosfamide (MAP/I) or mifamurtide ${ }^{2}$ (MAP/I +/- MTP) might eradicate micrometastatic disease, it does not cure unresectable disease. Several second-line and furtherline treatments have been tested, ${ }^{6-12}$ but have only showed marginal activity at best. Responses were achieved in $3 \%^{11}$ to $29 \%^{7-9}$ of patients with a median progression-free survival (PFS) ranging from $1 \cdot 4^{11}$ to about 4 months. ${ }^{8,9}$
Increased knowledge of the oncogenic pathways in high-grade osteosarcoma pathogenesis and the advent of targeted therapies have prompted drug exploration aimed at identifing key proteins. Consequently, small molecule inhibitors (imatinib) and monoclonal antibodies (eg. trastuzumab, bevacizumab, ${ }^{13}$ and drugs targeting IGF-1R $R^{13,14}$ have all been tested for activity. Although these compounds did not show significant activity in patients with advanced high-grade osteosarcoma, P-ERK1/2 was shown to have a a role in high-grade osteosarcoma growth, survival, neo-angiogenesis, and metastatic potential. ${ }^{15,16}$ Sorafenib, a multikinase inhibitor used in renal, hepatic, and thyroid cancers, ${ }^{17}$ prevented growth and metastasis in various osteosarcoma cell lines, in both in vitro and in vivo models. ${ }^{15}$ These findings led to a phase 2 trial to explore the activity of sorafenib in patients with relapsed, unresectable high-grade osteosarcoma after standard treatment. ${ }^{18}$ In this setting, sorafenib showed signs of antitumour activity in terms of the proportion of patients 
achieving a response (14\%), reduction of both 18F-fluorodeoxyglucose uptake and tumour density, and in improving pain control. Unfortunately, these encouraging results were short-lived, with $46 \%$ of patients progressionfree at 4 months and $29 \%$ progression-free at 6 months. ${ }^{18}$

We subsequently showed that the AKT-mTOR pathway has a role in resistance to sorafenib in high-grade osteosarcoma. $^{19}$ Indeed, whereas sorafenib inhibits mTORC1 complex activity, sorafenib activates the mTORC2 complex and promotes tumour progression. Preclinical studies showed that the combination of sorafenib with the mTOR inhibitor everolimus ${ }^{19}$ effectively overcame this resistance mechanism. In phase $1 / 2$ trials ${ }^{20-22}$ this combination has been extensively studied in renal and hepatic cancers at several doses, showing its feasibility.

Therefore, we designed a non-randomised phase 2 trial to investigate the activity of sorafenib in combination with everolimus in patients with inoperable high-grade osteosarcoma that had progressed after standard chemotherapy treatment.

\section{Methods \\ Patients and study design}

Patients with histologically documented high-grade osteosarcoma that was either unresectable or locally advanced or metastatic, and had progressed after first-line or second-line treatments were enrolled at one of three Italian Sarcoma Group centres. Eligibility criteria included: progressive and measurable disease according to Response Evaluation Criteria In Solid Tumors (RECIST 1.1; bone lesions were permitted), ${ }^{23} 18$ years of age or older, Eastern Cooperative Oncology Group (ECOG) performance status of 0 or 1 , life expectancy of 3 months or longer, and adequate liver function (defined as total bilirubin $\leq 1.5$ times the institutional upper limit of normal [ULN]; aspartate aminotransferase and alanine aminotransferase $\leq 2.5$ times ULN [ $\leq 5$ times ULN in presence of liver metastases]; international normalised ratio for prothrombin time $\leq 1.5$ times ULN), adequate renal function (serum creatinine $\leq 2$ times ULN), and adequate bone marrow function (haemoglobin $\geq 90 \mathrm{~g} / \mathrm{L}$, absolute neutrophil count $\geq 1.5 \times 10^{9}$ cells per $\mathrm{L}$, platelet count $\geq 100 \times 10^{9}$ cells/L). In particular, all patients were assessed by the sarcoma board that included a thoracic surgeon with at least 10 years' experience in sarcoma. Patients with exclusively lung metastases were carefully assessed for eligibility for metastasectomy. In this trial, we included only those deemed non-resectable. We excluded patients with severe or uncontrolled medical disorders ( $\geq$ grade 2 of Common Terminology Criteria for Adverse Events version 4.03 [CTCAE version 4.03]) that could jeopardise the outcomes of the study. The appendix shows details on inclusion and exclusion criteria (appendix pp 1-2).

The institutional review board and independent ethics committee of each participating centre revised and approved the protocol. The trial was done according to the

Declaration of Helsinki and the International Conference on Harmonization of Good Clinical Practice guidelines. All patients provided written informed consent before enrolment after being informed about the purpose, the expected risks, and the investigational nature of the study.

\section{Procedures}

Patients took $400 \mathrm{mg}$ sorafenib twice a day together with $5 \mathrm{mg}$ everolimus once a day. Researchers and participants were not masked to drug assignments, and the study ran until disease progression, unacceptable toxic effects, or patient refusal. Other predefined reasons for patient removal from the trial were the following: investigator's decision, substantial non-compliance with study requirements, pregnancy, use of illicit drugs or other prohibited substances, development of concurrent illness which could jeopardise clinical status and trial endpoints, or interruption of study drugs for more than 30 days.

Chest and abdomen CT or MRI were done at baseline, and repeated every 2 months if clinically indicated. 18FDG-PET scan was suggested but not mandatory and was done at baseline, during the third week of treatment, and then again if clinically indicated. Adverse events were assessed and graded according to CTCAE version 4.03. We managed adverse events with predefined rules (appendix p 3-9). In case of grade 3 or 4 neutropenia, thrombocytopenia, or skin toxicity (especially hand-foot skin reaction), sorafenib and everolimus were reduced by one dose level (600 mg sorafenib and $2.5 \mathrm{mg}$ everolimus daily) or by two dose levels (400 mg sorafenib once a day and $2.5 \mathrm{mg}$ everolimus every other day). Whenever feasible, patients were put back on the higher dose. If the adverse event did not resolve by suspending treatment, we removed patients from the trial. If grade 2 mucositis arose, both drugs were reduced by one dose level. If

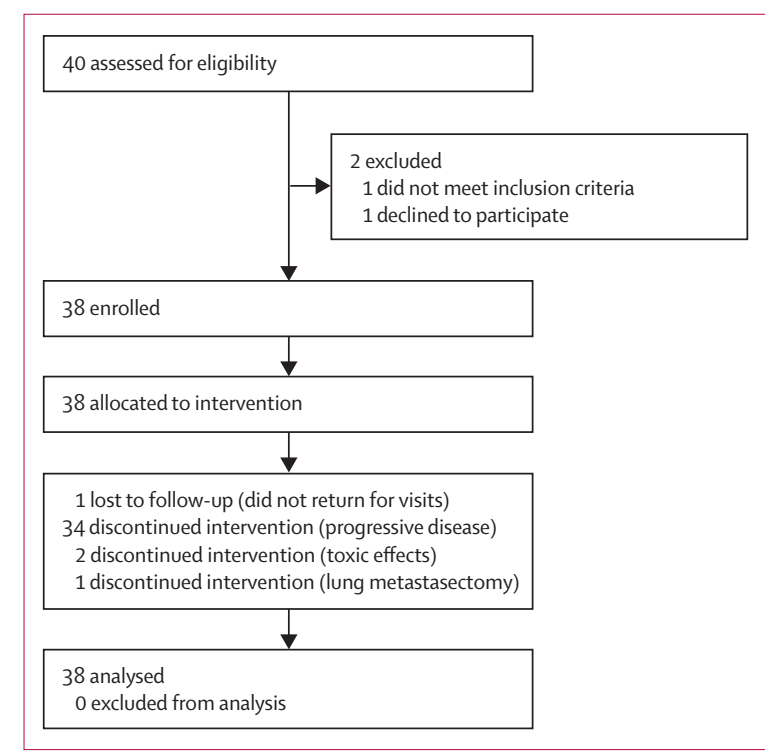

Figure 1: Trial profile
See Online for appendix

For the protocol see http://www. tumoriraripiemonte.it/public/ ISG\%20SERIO\%20Trial\%20V\%20 2.2.pdf 


\begin{tabular}{|c|c|}
\hline & $\mathrm{N}$ \\
\hline Patients & $38(100 \%)$ \\
\hline Age (years) & $31(18-64)$ \\
\hline \multicolumn{2}{|l|}{ Sex } \\
\hline Men & $23(61 \%)$ \\
\hline Women & $15(39 \%)$ \\
\hline \multicolumn{2}{|l|}{ Metastatic at diagnosis } \\
\hline Yes & $9(24 \%)$ \\
\hline No & $29(76 \%)$ \\
\hline \multicolumn{2}{|c|}{ High-grade osteosarcoma histotypes } \\
\hline Osteoblastic & $27(71 \%)$ \\
\hline Chondroblastic & $8(21 \%)$ \\
\hline Fibroblastic & $2(5 \%)$ \\
\hline Telangiectatic & $1(3 \%)$ \\
\hline \multicolumn{2}{|c|}{ ECOG performance status at start } \\
\hline 0 & $16(42 \%)$ \\
\hline 1 & $20(53 \%)$ \\
\hline $2^{*}$ & $2(5 \%)$ \\
\hline \multicolumn{2}{|l|}{ Lactate dehydrogenase $>$ ULN } \\
\hline No & $20(53 \%)$ \\
\hline Yes & $18(47 \%)$ \\
\hline \multicolumn{2}{|l|}{ Alkaline phosphatase $>$ ULN } \\
\hline No & $16(42 \%)$ \\
\hline Yes & $22(58 \%)$ \\
\hline \multicolumn{2}{|c|}{ Lines of chemotherapy after MAP/I } \\
\hline 1 & $2(5 \%)$ \\
\hline$>1$ & $36(95 \%)$ \\
\hline \multicolumn{2}{|l|}{ Necrosis after MAP/I } \\
\hline Poor $(<90 \%)$ & $24(63 \%)$ \\
\hline $\operatorname{Good}(\geq 90 \%)$ & $10(27 \%)$ \\
\hline Not available or missing & $4(10 \%)$ \\
\hline Previous surgery & $2(0-7)$ \\
\hline 0 & $1(3 \%)$ \\
\hline 1 & $6(16 \%)$ \\
\hline 2 & $16(42 \%)$ \\
\hline$>2$ & $15(39 \%)$ \\
\hline \multicolumn{2}{|l|}{ Sites of metastases } \\
\hline Lung only & $12(32 \%)$ \\
\hline Lung and bone or viscera & $22(58 \%)$ \\
\hline Bone only & $4(10 \%)$ \\
\hline \multicolumn{2}{|l|}{ Analgaesic use } \\
\hline None & $13(34 \%)$ \\
\hline NSAIDs or acetaminophen & $5(13 \%)$ \\
\hline Steroids & $3(8 \%)$ \\
\hline Mild narcotics & $7(18 \%)$ \\
\hline Strong narcotics & $10(27 \%)$ \\
\hline \multicolumn{2}{|c|}{$\begin{array}{l}\text { Data are n (\%) or median (range). ECOG=Eastern Cooperative Oncology } \\
\text { Group. ULN=Upper limit of normal. MAP/l=methotrexate doxorubicin, cisplatin } \\
+ \text { +- ifosfamide. NSAIDs=non-steroidal anti-inflammatory drugs.. }{ }^{*} \text { Only patients } \\
\text { with EOCG performance status } 2 \text { for orthopaedic problems were eligible. }\end{array}$} \\
\hline
\end{tabular}

hypertension or diarrhoea were not controlled by concomitant drugs, sorafenib was reduced by one dose level or temporarily suspended. Hyperglycaemia or hyperlipidaemia of grade 1 toxicity or greater was treated with appropriate pharmaceutical treatment (ie, oral antidiabetic, insulin, or statins) whenever feasible rather than by dose reduction. In case of creatinine increase, febrile neutropenia or any other clinically relevant unexpected toxic effects, both drugs were stopped until resolution.

\section{Outcomes}

The primary endpoint of the trial was 6 month PFS. We assessed the following secondary endpoints: overall PFS; overall survival; the proportion of patients who had an overall response that was defined as complete responses, partial responses and minor responses (ie, lesions shrunk $<30 \%$ but $>10 \%$ in the sum of lesion widest diameters, so below the threshold of partial response); the proportion of patients who achieved disease control (overall response rate and stable diseases); duration of response; pain improvement; and safety. RECIST responses had to be confirmed after at least four weeks. On paraffin-embedded tumour specimens, immunohistochemical expression of P-ERK1/2 and P-RPS6 was assessed to explore whether expression was associated with outcome, and scored as follows: less than $10 \%$ positive cells: $0+; 10-50 \%$ positive cells: $1+$; more than $50 \%$ positive cells, and high staining intensity: $2+$.

We calculated PFS from trial entry until progression, unacceptable toxic effects, or death, whichever came first. We calculated overall survival from trial entry until death. We calculated duration of response from first nonprogression assessment until either progression or death. In the absence of an event or loss to follow-up, all survival endpoints were censored on the last date the patient was known to be event free.

We assessed any sign of tumour-related pain improvement by the Pain and Analgesic Score (PAS) ${ }^{24}$ and the Brief Pain Inventory (BPI) short form score. ${ }^{25}$ PAS tracked the use of painkillers attributing an increasing value to each of them (eg, use of acetaminophen scores 1 point whereas morphine scores 4). This scale has the strength to be objective but it does not quantify each drug dose. The BPI score recorded changes in patient symptoms and is a selfadministered questionnaire; thus, it has the inherent limit of patient compliance to repeatedly completing questionnaires. To understand the real effect of study drugs on symptoms, we deemed assessable only those patients who had completed at least three forms (assessment at baseline, at least one during treatment and at the off-treatment visit).

\section{Statistical analysis}

There is a surprising lack of published data for PFS of patients with high-grade osteosarcoma that have relapsed after standard treatments. At the same time, reported objective responses to chemotherapy after MAP/I vary widely, making this endpoint unsuitable in 
the context of patients with advanced and unresectable high-grade osteosarcoma. Therefore, we chose PFS at 6 months as a primary endpoint rather than objective response and used the 6 month PFS recorded in our previous trial ${ }^{18}$ as a point of reference. Thus, the trial was designed to discard a 6-month PFS of $25 \%$ (null hypothesis) aiming to reach a 6 -month PFS of $50 \%$ or higher (alternative hypothesis). Using Simon's optimum two-stage design ${ }^{26}$ and setting $\alpha$-error at 0.05 and $\beta$-error at $0 \cdot 10$, the presence of at least six successes in the 17 patients enrolled in the first stage allowed the trial to proceed to the second stage in which 20 more patients were needed to be enrolled for the minimum total of 37 patients.

The presence of 14 or more successes (ie, patients alive at 6 months without progression) allowed the experimental treatment to be regarded as worth further study. The intention-to-treat analysis included all patients who received at least one dose of each drug. The population assessable for treatment activity comprised all patients for whom at least one disease assessment (either clinical or radiological) was done. The primary endpoint was analysed in the intention-to-treat population. We estimated survival endpoints according to the KaplanMeier method, with 95\% CIs. RECIST overall responses and disease control were calculated and reported with 95\% CIs. We investigated the effect of P-ERK1/2 and P-RPS6 expression by comparing survival outcomes with the two-sided Mantle-Cox log-rank test, Fisher's exact test, and the Mantel-Haenszel odds ratio (OR) estimate. We compared baseline versus on-treatment PAS and BPI scores with a paired student's $t$ test. We computed all statistics with IBM SPSS Statistics (version 20) and GraphPad Prism (version 5).

This trial is registered with ClinicalTrials.gov, number NCT01804374.

\section{Role of the funding source}

The funder sponsored the trial through an unrestricted grant from Bayer and everolimus was supplied by Novartis. Pharmaceutical companies had no role in data collection and interpretation, or writing of the report. The funder of the study was involved in the scientific evaluation of the study rationale, in patient referral to enrolling centres and data collection, but had no role in study design, data analysis, data interpretation, or writing of the report. All authors had access to the raw data, vouch for the completeness and accuracy of the data and analyses and approved the final version of the manuscript. The corresponding author had final responsibility for the decision to submit for publication.

\section{Results}

From June 16, 2011, to June 4, 2013, we enrolled 38 patients. To be eligible, patients had to be inoperable and to have progressed after at least standard therapy (MAP/I) (figure 1; appendix). Two patients signed the

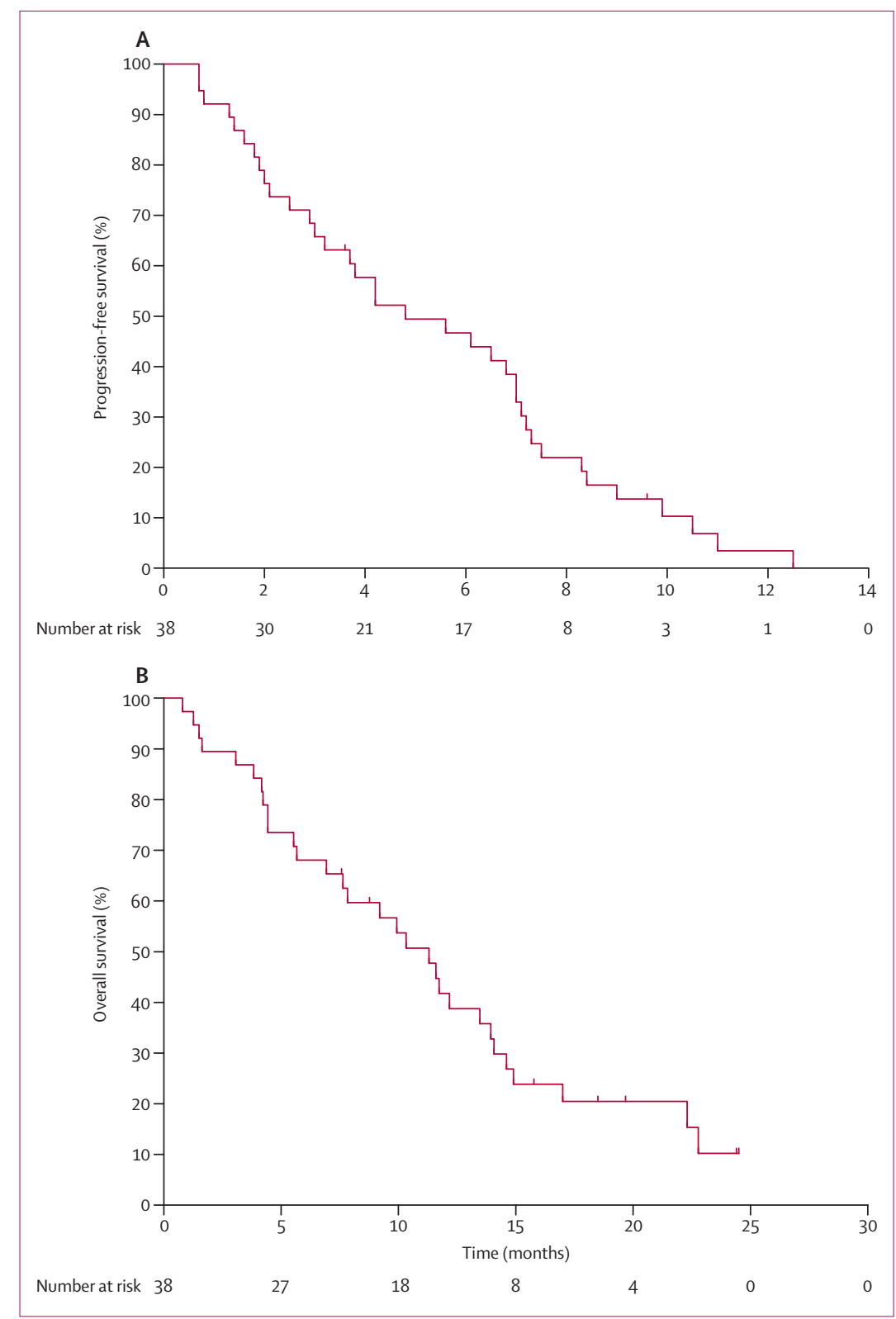

Figure 2: Kaplan-Meier plots for progression-free survival (A) and overall survival (B) in all patients (intention-to-treat analysis)

informed consent the same day and as we considered it unethical to exclude one of these patients from the trial, we enrolled 38 patients rather than the 37 planned. Table 1 describes patient characteristics at baseline. All patients had already received MAP/I chemotherapy; the median number of previous systemic regimens was two (range 1-3). All patients were treated according to the protocol and were included in the safety and activity analyses. All analyses were done after the last patient had been followed up for at least 6 months.

After completion of stage 1 , nine (53\%) of 17 patients were progression-free at 6 months. Therefore, we enrolled 


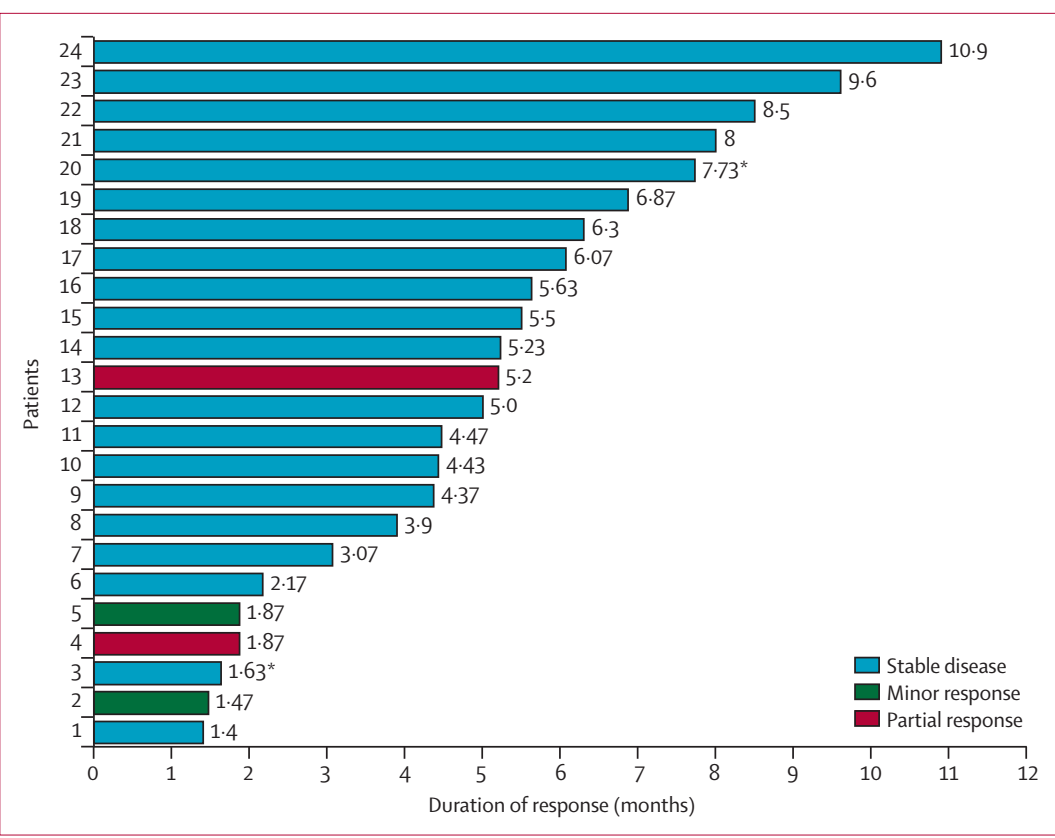

Figure 3: Duration of response

14 patients were not included in the plot because they progressed at the time of first assessment. ${ }^{*}$ Censored.

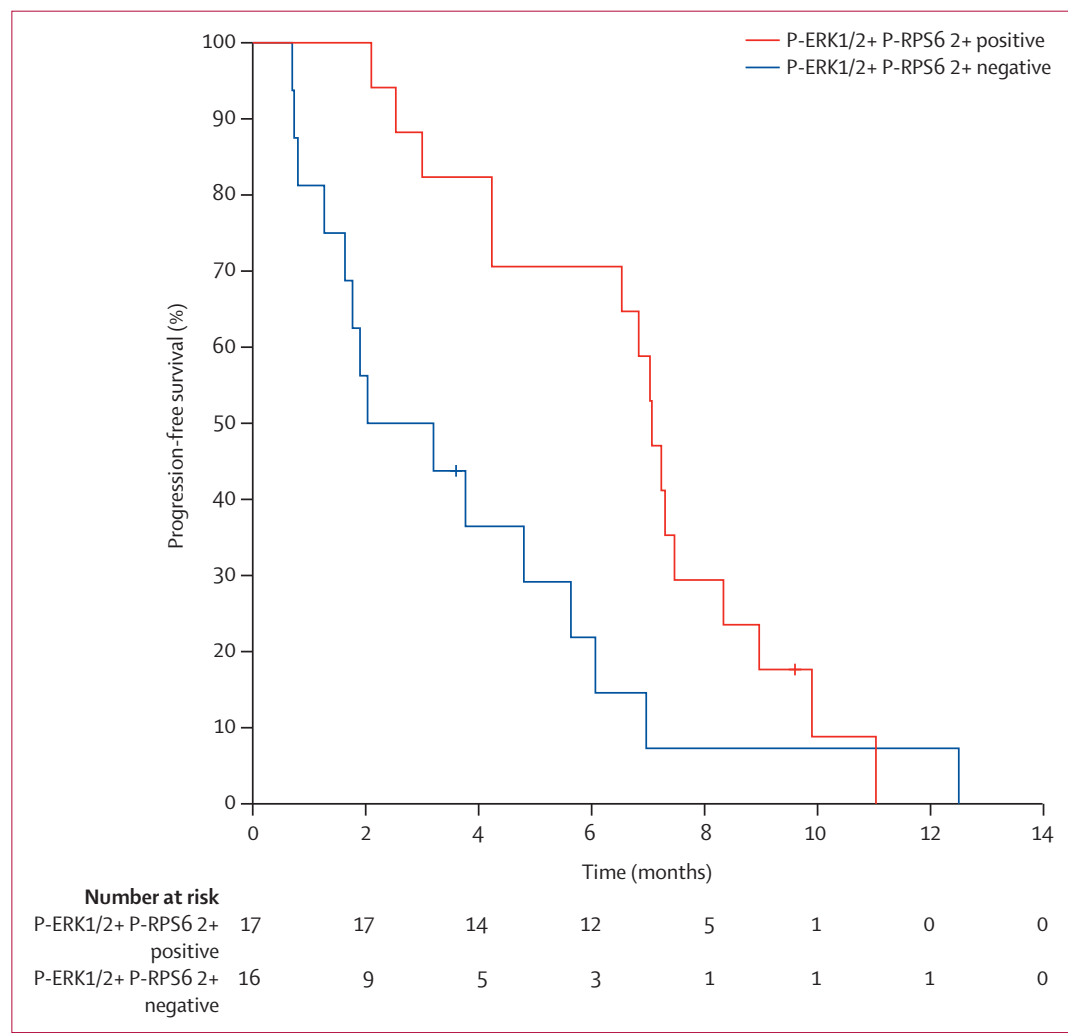

Figure 4: Kaplan-Meier plot for progression-free survival based on P-ERK1/2 and P-RPS6 immunohistochemical expression

Progression-free survival curves for patients positive for both P-ERK1/2 and P-RPS6 expression versus P-ERK1/2 and P-RPS6 negative patients. Log-rank test $\mathrm{p}=0.021$. Crosses indicate censoring. a further 21 patients for stage 2 and 17 (45\%) of 38 patients were free from progression at 6 months (figure 2). Median PFS was 5 months (95\% CI 2-7) with eight (21\%) of 38 patients receiving sorafenib and everolimus for 8 months or more. Patients received treatment for a median of 10 months (range 8-12 months). 24 (63\%) of 38 patients were free from disease progression after 2 months and had a median duration of response of 5 months (95\% CI 4-6). Figure 3 shows duration of response.

Three patients with partial or minor response withdrew from the study before 4 months because of toxic effects including one case of recurrent hand-foot syndrome plus skin rash (despite dose reduction and interruption) and two cases of disease progression (detected by CT scan undertaken as per protocol). Treatment was terminated owing to: toxic effects in two (5\%) patients; disease progression in $34(89 \%)$ patients and lung metastasectomy in one $(3 \%)$ patient. One $(3 \%)$ patient was lost to followup. Two patients were censored. The first censored patient was lost to follow-up after leaving Italy and was censored at 3.6 months at her last clinical assessment (without evidence of progression); the second censored patient underwent lung metastasectomy in the absence of disease progression after nearly 10 months of treatment. After surgery, disease was no longer assessable and we censored this patient at the day before surgery. The median followup of surviving patients was 10 months (95\% CI 6-14). Median overall survival was 11 months (95\% CI 8-15) with $14(37 \%)$ of 38 patients alive after 12 months and two (5\%) alive after 24 months (figure 2). No deaths were deemed related to experimental treatment.

Of the 38 enrolled patients, we recorded two (5\%) partial responses and two minor responses $(5 \%)$, which resulted in an overall response being achieved in $10 \%$ of patients (95\% CI 0.3-21; figure 3). We did not note any complete responses. 20 (53\%) patients achieved stable disease whereas 14 (37\%) of 38 patients had disease progression. Disease control was achieved in 24 (63\%) of 38 patients and median treatment duration lasted 5 months (IQR $2-7)$. We noted ten (33\%) non-dimensional responses (ie, CT-PET responses without tumour shrinkage) with 18 FDG PET in the 30 patients who had PET scans (mean 52\% [SD $26 \%$ reduction in standardised uptake value in responding patients). One patient with stable disease underwent lung metastasectomy after 10 months. To understand the clinical meaning of disease stability, if any, we estimated PFS for the entire patient cohort according to the last treatment received before enrolment (either surgery, chemotherapy, or radiotherapy). Median PFS was 2 months (95\% CI 1-3), which shows the aggressiveness of underlying high-grade osteosarcoma. The pathology report described four metastatic nodules in this patient of high-grade osteoblastic and chondroblastic osteosarcoma. Necrosis in these nodules ranged from $10 \%$ to $60 \%$ in the nodules (average 30-40\% localised mostly in the centre of each metastasis). 
We tracked and recorded self-perceived improvement in pain management. We did not record a reduction of analgesic use in terms of PAS score (mean baseline 1.9 [SD 1.7]; mean best on treatment 1.8 [SD 1.7]; $\mathrm{p}=0 \cdot 619$ ). We recorded an improvement in 22 (58\%) of 38 patients fully assessable by BPI questionnaire (mean baseline score: 36 points [SD 19]; mean best on treatment score: 24 [SD 16]; $\mathrm{p}=0 \cdot 004)$. Off-treatment and on-treatment visit forms were not available in 11 and five patients, respectively.

Immunohistochemical expression of P-ERK1/2 was fully assessable in 33 (87\%) of 38 patients and immunohistochemical expression of P-RPS6 in 35 (92\%) patients. We did not do high-quality immunohistochemistry for P-ERK1/2 in five high-grade osteosarcoma samples and for P-RPS6 in three high-grade osteosarcoma samples because of a technical problem probably arising from prolonged decalcification that impaired sample antigenicity. 20 (61\%) of 33 assessable tumour specimens had positive P-ERK1/2 staining and this was significantly associated with a greater probability of being progression-free at 6 months: 12 $(60 \%)$ of 20 P-ERK1/2 positive patients vs three (23\%) of $13 \mathrm{P}$-ERK1/2 negative patients were progression-free at 6 months (OR 5, 95\% CI 1.04-24.03; p=0.045). Positive P-RPS6 staining (score 2+) was noted in 17 (49\%) of 35 assessable specimens and was similarly associated with a greater probability of being progression-free at 6 months: $17(61 \%)$ of 28 P-RPS6 positive patients $v s$ none of the 7 P-RPS6 negative patients were progression-free at 6 months $(p=0 \cdot 008$; OR not assessable). Positive staining of both P-ERK1/2 and P-RPS6 were seen in 17 (51\%) of 33 patients and predicted a better median PFS (7 months [95\% CI 7-8] for double positive patients vs 2 months [95\% CI 0-5] for non-double positive patients]; $\mathrm{p}=0.021$ ) with a higher probability of PFS at 6 months (12 [71\%] of 17 vs three [19\%] of 16 , OR $10 \cdot 4,95 \%$ CI $2 \cdot 03-53 \cdot 2 ; p=0 \cdot 005$; figure 4). The lung metastases removed in one patient had low levels of both P-ERK1/2 and P-RPS6 expression.

The median follow-up for the safety analysis was 6 months (IQR 3-8). At final follow-up, no patient remained on treatment. We recorded at least one adverse event in all 38 patients. $43(10 \%)$ of 424 adverse events were grade 3-4 (table 2 ). The most common grade 3-4 adverse events were the following: lymphopenia (six [16\%] of 38 patients), hypophosphatemia (six [16\%]), hand-foot syndrome (five [13\%]), thrombocytopenia (four [11\%]), fatigue (two [5\%]), oral mucositis (two [5\%]), diarrhoea (two [5\%]), and anaemia (two [5\%]; table 2). All of these adverse events were causally related to the study drugs. One (3\%) patient had a grade 3 pneumothorax that required trans-thoracic drainage, which recurred at the time of progressive disease. In both instances, the events were regarded as a serious adverse event related to the study drugs. We reported no other serious adverse events during the trial. No deaths were related to the

\begin{tabular}{|c|c|c|c|c|c|}
\hline & All (\%) & Grade $1(\%)$ & Grade 2 (\%) & Grade $3(\%)$ & Grade $4(\%)$ \\
\hline Thrombocytopenia & 22 & $11(29 \%)$ & $7(18 \%)$ & $4(11 \%)$ & $0(0 \%)$ \\
\hline Anaemia & 19 & $14(37 \%)$ & $3(8 \%)$ & $2(5 \%)$ & $0(0 \%)$ \\
\hline Lymphopenia & 14 & $7(18 \%)$ & $1(3 \%)$ & $3(8 \%)$ & $3(8 \%)$ \\
\hline Leucopenia & 12 & $8(21 \%)$ & $3(8 \%)$ & $1(3 \%)$ & $0(0 \%)$ \\
\hline Neutropenia & 10 & $6(16 \%)$ & $3(8 \%)$ & $1(3 \%)$ & $0(0 \%)$ \\
\hline Febrile neutropenia & 1 & $0(0 \%)$ & $0(0 \%)$ & $1(3 \%)$ & $0(0 \%)$ \\
\hline Oral mucositis & 20 & $11(29 \%)$ & $7(18 \%)$ & $2(5 \%)$ & $0(0 \%)$ \\
\hline Diarrhoea & 18 & $5(13 \%)$ & $11(29 \%)$ & $2(5 \%)$ & $0(0 \%)$ \\
\hline Hypophosphataemia & 18 & $5(13 \%)$ & $7(18 \%)$ & $6(16 \%)$ & $0(0 \%)$ \\
\hline Fatigue & 16 & $8(21 \%)$ & $6(16 \%)$ & $2(5 \%)$ & $0(0 \%)$ \\
\hline Hypercholesterolaemia & 15 & $14(37 \%)$ & $1(3 \%)$ & $0(0 \%)$ & $0(0 \%)$ \\
\hline Nausea & 14 & $10(26 \%)$ & $3(8 \%)$ & $1(3 \%)$ & $0(0 \%)$ \\
\hline Hypokalaemia & 14 & $10(26 \%)$ & $3(8 \%)$ & $1(3 \%)$ & $0(0 \%)$ \\
\hline Hypertriglyceridaemia & 14 & $9(24 \%)$ & $5(13 \%)$ & $0(0 \%)$ & $0(0 \%)$ \\
\hline Weight loss & 13 & $8(21 \%)$ & $4(11 \%)$ & $1(3 \%)$ & $0(0 \%)$ \\
\hline Aminotransferase increase & 12 & $10(26 \%)$ & $2(5 \%)$ & $0(0 \%)$ & $0(0 \%)$ \\
\hline Hyperglycaemia & 11 & $9(24 \%)$ & $2(5 \%)$ & $0(0 \%)$ & $0(0 \%)$ \\
\hline Abdominal cramps & 11 & $7(18 \%)$ & $4(11 \%)$ & $0(0 \%)$ & $0(0 \%)$ \\
\hline CK increase & 10 & $8(21 \%)$ & $1(3 \%)$ & $1(3 \%)$ & $0(0 \%)$ \\
\hline Infection & 9 & $1(3 \%)$ & $7(18 \%)$ & $1(3 \%)$ & $0(0 \%)$ \\
\hline Hypomagnesaemia & 9 & $9(24 \%)$ & $0(0 \%)$ & $0(0 \%)$ & $0(0 \%)$ \\
\hline Vomiting & 8 & $7(18 \%)$ & $1(3 \%)$ & $0(0 \%)$ & $0(0 \%)$ \\
\hline GGT increase & 8 & $4(11 \%)$ & $4(11 \%)$ & $0(0 \%)$ & $0(0 \%)$ \\
\hline Constipation & 8 & $6(16 \%)$ & $2(5 \%)$ & $0(0 \%)$ & $0(0 \%)$ \\
\hline Myalgia/arthralgia & 6 & $2(5 \%)$ & $3(8 \%)$ & $1(3 \%)$ & $0(0 \%)$ \\
\hline Hypertension & 6 & $4(11 \%)$ & $1(3 \%)$ & $1(3 \%)$ & $0(0 \%)$ \\
\hline Bilirubin increase & 5 & $4(11 \%)$ & $1(3 \%)$ & $0(0 \%)$ & $0(0 \%)$ \\
\hline Amylase or lipase increase & 4 & $4(11 \%)$ & $0(0 \%)$ & $0(0 \%)$ & $0(0 \%)$ \\
\hline Creatinine increase & 3 & $1(3 \%)$ & $2(5 \%)$ & $0(0 \%)$ & $0(0 \%)$ \\
\hline Cough & 3 & $3(8 \%)$ & $0(0 \%)$ & $0(0 \%)$ & $0(0 \%)$ \\
\hline Headache & 2 & $2(5 \%)$ & $0(0 \%)$ & $0(0 \%)$ & $0(0 \%)$ \\
\hline Ejection fraction decrease & 2 & $2(5 \%)$ & $0(0 \%)$ & $0(0 \%)$ & $0(0 \%)$ \\
\hline Bleeding & 2 & $2(5 \%)$ & $0(0 \%)$ & $0(0 \%)$ & $0(0 \%)$ \\
\hline Dysphagia & 1 & $0(0 \%)$ & $0(0 \%)$ & $1(3 \%)$ & $0(0 \%)$ \\
\hline Pneumothorax & 1 & $0(0 \%)$ & $0(0 \%)$ & $1(3 \%)$ & $0(0 \%)$ \\
\hline Hand-foot skin reaction & 27 & $9(24 \%)$ & $13(34 \%)$ & $5(13 \%)$ & $0(0 \%)$ \\
\hline Rash & 24 & $18(47 \%)$ & $5(13 \%)$ & $1(3 \%)$ & $0(0 \%)$ \\
\hline Acneiform eruption & 16 & $14(37 \%)$ & $1(3 \%)$ & $1(3 \%)$ & $0(0 \%)$ \\
\hline Xerosis & 11 & $11(29 \%)$ & $0(0 \%)$ & $0(0 \%)$ & $0(0 \%)$ \\
\hline Pruritus & 5 & $5(13 \%)$ & $0(0 \%)$ & $0(0 \%)$ & $0(0 \%)$ \\
\hline
\end{tabular}

experimental treatment; all deaths were attributed to disease progression.

We had to reduce or temporarily suspend study drugs in $25(66 \%)$ of 38 patients because of toxic effects (table 3 ). Generally, we deemed short drug interruptions as useful for recovery from toxic effects in 22 (58\%) of 38 patients, and these arose mostly during the first treatment month. The frequency of administered dose of sorafenib was $77 \%$ of planned, and $82 \%$ for everolimus. The mean 


\begin{tabular}{|c|c|}
\hline & Number (\%) or time \\
\hline $\begin{array}{l}\text { Treatment permanently interrupted because of } \\
\text { toxic effects }\end{array}$ & $2(5 \%)$ \\
\hline $\begin{array}{l}\text { Treatment dose reduced/temporarily } \\
\text { interrupted }\end{array}$ & $25(66 \%)$ \\
\hline Treatment temporarily interrupted & $22(58 \%)$ \\
\hline \multicolumn{2}{|l|}{ Dose of sorafenib given } \\
\hline 800 mg per day & $19(50 \%)$ \\
\hline $600 \mathrm{mg}$ per day ( -1 dose level) & $6(16 \%)^{*}$ \\
\hline 400 mg per day ( -2 dose level) & $19(50 \%)$ \\
\hline \multicolumn{2}{|l|}{ Days sorafenib held for one interruption } \\
\hline Mean & $7(4)$ \\
\hline Median & $6(4-9)$ \\
\hline \multicolumn{2}{|l|}{ Days sorafenib held during trial } \\
\hline Mean & $24(20)$ \\
\hline Median & $15(10-36)$ \\
\hline Dose of everolimus given $5 \mathrm{mg}$ per day & $25(66 \%)$ \\
\hline 2.5 mg per day ( -1 dose level) & $13(34 \%)$ \\
\hline $2.5 \mathrm{mg}$ every other day ( -2 dose level) & $0(0 \%)$ \\
\hline \multicolumn{2}{|l|}{ Days everolimus held for one interruption } \\
\hline Mean & $7(5)$ \\
\hline Median & $5(4-9)$ \\
\hline \multicolumn{2}{|l|}{ Days everolimus held during trial } \\
\hline Mean & $27(21)$ \\
\hline Median & $17(12-45)$ \\
\hline \multicolumn{2}{|c|}{ 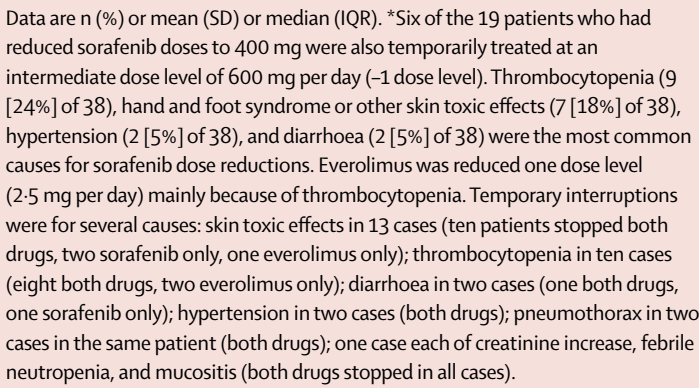 } \\
\hline
\end{tabular}

temporary interruption duration was 7 days [SD 4] for sorafenib and 7 days [SD 5] for everolimus (table 3).

\section{Discussion}

In this phase 2 non-randomised trial, we assessed the activity of the combination of sorafenib and everolimus in patients with high-grade osteosarcoma progressing after standard multidisciplinary treatment. Toxic effects were consistent with those reported in previous phase 1 studies ${ }^{20-22}$ of the same combination and confirmed the treatment feasibility. In terms of activity, $45 \%$ of the patients were free from progression at 6 months. Finally, the immunohistochemical expression of P-ERK1/2 and P-RPS6 was associated with a better response to study drugs.

The rationale for this combination was built on our previous preclinical data showing that inhibition of the
mTOR pathway with everolimus ${ }^{19}$ might increase the activity of sorafenib (panel). As the primary endpoint, we deliberately set the PFS rate high (at 50\%) and of quite long duration (6 months) because findings of our previous trial had already shown 29\% 6-month PFS with sorafenib alone. ${ }^{18}$ In theory, a Bayesian statistical design might have allowed a less stringent assumption. However, although attractive, the Bayesian statistical approach has seldom been used in non-randomised trials addressing one histotype. ${ }^{31}$ Our trial achieved a 45\% 6-month PFS, which is less than the prespecified threshold of activity to deem the combination of sorafenib and everolimus worthy of a phase 3 trial. Nevertheless, in the context of unresectable or relapsed high-grade osteosarcoma, our results compare favourably with results of other published studies addressing patient cohorts with similar clinical characteristics (appendix p 10-11), and our results are higher than the widely accepted 3-month and 6-month PFS benchmark values of $40 \%$ and $20 \%$, respectively, ${ }^{30}$ for a drug to be regarded as active in soft-tissue sarcomas. ${ }^{28}$ Similarly, our findings compare well with the median PFS of 1.8 months and with the 6 month PFS of less than $10 \%$ in paediatric patients affected by relapsed high-grade osteosarcoma who failed to achieve a second CR reported by Leary and colleagues, ${ }^{29}$ although such cross-trial comparisons should be made with caution. Finally, our outcome needs to be considered within the context of the very unfavourable subset (inoperable, median age 31 years, and heavily pre-treated $)^{32}$ to which our patients belonged. Thus, although this combination treatment did not modify patient final outcome, the reported activity data might contribute to the development of molecular approaches for treatment and appropriate patient selection.

We chose PFS rather than objective response as our primary endpoint because tumour shrinkage in osteosarcoma is difficult to obtain and detect. Assessment of the activity of targeted therapy is always challenging, and more so in osteosarcoma where either calcification or necrosis can arise in the absence of tumour shrinkage ${ }^{18}$ even at disease onset. Furthermore, bone lesion response assessment is demanding in any tumour. ${ }^{23}$ The proportion of patients achieving an overall response could be perceived as unsatisfactory, but this result is within the same range as those recorded in breast cancer, ${ }^{33}$ renal cell carcinoma, ${ }^{34,35}$ and advanced pancreatic neuroendocrine tumours. ${ }^{36}$ Moreover, stable disease has been related to tumour control and treatment benefit. ${ }^{37}$ Modified RECIST criteria ${ }^{38}$ and improved metrics are required to assess tumour response to tyrosine-kinase inhibitor treatments as reported by Choi and colleagues. ${ }^{39}$ Both criteria suggest that a tumour shrinkage of greater than $10 \%$ might be significant. Indeed, previous last-line median PFS was very short.

To improve the interpretation of imaging findings, we did 18FDG-PET scans. PET responses cannot be 
compared with responses reported in previous studies. However, because all enrolled patients had confirmed progression at trial entry, long-lasting disease stability is less likely to be due to disease dormancy.

The reported adverse events are consistent with those described in phase 1 trials. Notwithstanding, our cohort was younger than the ones enrolled in those trials and tolerated the drug combination slightly better than what has been previously reported. ${ }^{20-22}$ Variable drug dosages and clinical settings may explain some of the differences recorded. Generally, drug-related adverse events were quite common and needed close contact between clinician and patient to attain the best management. As anticipated, increased toxic effects from sorafenib and everolimus overlapped in the form of skin toxicity, stomatitis, thrombocytopenia, and fatigue. In particular, fatigue might be a distressing adverse event especially in the chronic setting. Although clinically significant, in this young and highly motivated population, fatigue never mandated treatment discontinuation. Short interruptions and dose modulations helped to permit drug reintroduction and prolonged use in responsive patients.

A strong rationale exists to combine a multikinase inhibitor targeting tyrosine kinase receptors, such as PDGFR, VEGFR, and ERK1/240 with a selective inhibitor of mTOR. ${ }^{19,41}$ This prompted us to explore the clinical validity of phosphorylated-ERK1/2 and phosphorylated-RPS6 as predictive biomarkers of sorafenib and everolimus combination activity. Our results supported the decision to assess P-ERK1/2 and P-RPS6 because their expression was associated with a significant improvement in the response to the combination. Acknowledging the absence of a control group, our data are consistent with the predictive roles of both P-ERK1/2 and P-RPS6 in renal and hepatic cancers. ${ }^{42}$ However, we cannot definitively state whether these biomarkers are predictive or prognostic. Nonetheless, our data support the analytic validity of these biomarkers, which had been previously studied by several other groups on different series. ${ }^{13-15,18}$ Therefore, these biomarkers might be helpful for further trials on this or similar combinations in high-grade osteosarcoma and in other tumours.

The major limitation of our trial is the absence of a control group. We conceived this trial to rapidly determine whether preclinical results with sorafenib and everolimus were translatable to the clinical setting. A randomised trial design would have needed much more time to complete for such a rare tumour. However, we recognise that a randomised placebo-controlled trial would have allowed us to better understand the effect of everolimus on both overall toxic effects and the activity of the combination. For instance, it was challenging to attribute mucositis or skin toxic effects to either drug. However, we sought to strengthen the results by choosing objective and easily assessable endpoints for a fairly distant point in time to minimise the Hawthorne effect, ${ }^{43}$ and to generate clinically useful information about the activity of this combination. The challenge
Panel: Research in context

\section{Systematic review}

We searched PubMed for original research articles and reviews published in English before July 31, 2014, using the terms "osteosarcoma", "MAPK", "sorafenib", "mTOR", and combinations thereof. We found no clinical trial with combined terms "MAPK- and mTOR-pathway inhibitors in osteosarcoma", one clinical trial with sorafenib alone, ${ }^{18}$ two clinical trials with "mTOR inhibitor temsirolimus" when combined with different osteosarcoma agents, ${ }^{14,27}$ and six clinical trials addressing the feasibility of sorafenib and everolimus combinations at different dosages. ${ }^{20-22}$ We used preclinical data for the superiority of sorafenib and everolimus in combination over single agents ${ }^{19}$ to design our trial.

\section{Interpretation}

To our knowledge, this is the first trial exploring a combination of fully oral target treatments after failure of the most active chemotherapy drugs for osteosarcoma. In the context of a rapidly progressive disease, the observed 6-month progression-free survival (PFS) of $45 \%$ compares favourably with results reported by chemotherapies or targeted therapies. ${ }^{6-14,18,28,29}$ Moreover, this result is better than the widely accepted threshold for soft-tissue sarcoma drug activity ${ }^{28}\left(20 \%\right.$ for 6 -month PFS). ${ }^{30}$ Thus, this strategy should continue to be considered, either by modulating the same drugs or improving the inhibitory specificity with novel targeted therapies. Importantly, the immunohistochemical expression of phospho-ERK1/2 and phospho-RPS6 identified a subpopulation of patients who benefited most from the combination. These data suggest that any further clinical development of sorafenib and everolimus in combination should select patients on the basis of P-ERK1/2 and P-RPS6 expression, both in osteosarcoma and in other relevant tumour types.

of studying a young patient population with relapsed and inoperable high-grade osteosarcoma in a randomised way is confirmed by the absence of randomised trials in the advanced setting. Although our patients had a median age of 31 years, which is older than the expected peak incidence of osteosarcoma, ${ }^{3}$ they were young in terms of lifespan. Indeed, in such a cohort of patients, it would be difficult to propose a placebo controlled trial.

In conclusion, our trial shows that the combination of the sorafenib and everolimus has activity in patients with relapsed and inoperable high-grade osteosarcoma tumours. Nevertheless, the combination did not significantly affect the dismal prognosis of advanced high-grade osteosarcoma. Patients with overexpression of both P-ERK1/2 and P-RPS6 had the greatest benefit from the combination. Therefore, as we await significant advancement in the knowledge of osteosarcoma biology or innovative chemotherapy, any further trials targeting 
these pathways should consider selecting the experimental population on the basis of the proposed biomarkers. In a subset of patients with tumours expressing P-ERK1/2 and/or P-RPS6, this combination of sorafenib and everolimus might warrant further prospective controlled clinical trials.

\section{Contributors}

GG, SF, PP, and MA designed and developed the trial. GG, EP, VF, RB, AT, LD, RB, SDA, FF, PGC, SF, and MA were responsible for patien inclusion. GG, YP, SD, LD, EM and MG collected data. GG, YP, SD, FC and LD analysed and interpreted the data. GG and MA wrote the manuscript. All the authors reviewed the Article for intellectual content, provided comments, and gave final approval.

\section{Declaration of interests}

GG and MA have given expert testimony for Bayer. PGC has consulted/ advised and has received research funding from both Novartis and Bayer, and has received honoraria and other remuneration from Novartis. All other authors declare no relevant relationship to disclose.

\section{Acknowledgments}

This study was funded by the Italian Sarcoma Group. We thank all the patients and their families for participation in the present trial; Bayer for the unrestricted grant to Italian Sarcoma Group; Novartis for the supply of everolimus; and Joan C Leonard for language editing.

\section{References}

1 Gatta G, van der Zwan JM, Casali PG, et al, and the RARECARE working group. Rare cancers are not so rare: the rare cancer burden in Europe. Eur J Cancer 2011; 47: 2493-511.

2 Meyers PA, Schwartz CL, Krailo MD, et al, and the Children's Oncology Group. Osteosarcoma: the addition of muramyl tripeptide to chemotherapy improves overall survival--a report from the Children's Oncology Group. J Clin Oncol 2008; 26: 633-38.

3 Bielack SS, Kempf-Bielack B, Delling G, et al. Prognostic factors in high-grade osteosarcoma of the extremities or trunk: an analysis of 1,702 patients treated on neoadjuvant cooperative osteosarcoma study group protocols. J Clin Oncol 2002; 20: 776-90.

4 Kempf-Bielack B, Bielack SS, Jürgens H, et al. Osteosarcoma relapse after combined modality therapy: an analysis of unselected patients in the Cooperative Osteosarcoma Study Group (COSS). J Clin Oncol 2005; 23: 559-68.

5 Ferrari S, Briccoli A, Mercuri M, et al. Postrelapse survival in osteosarcoma of the extremities: prognostic factors for long-term survival. J Clin Oncol 2003; 21: 710-15.

6 Harris MB, Cantor AB, Goorin AM, et al. Treatment of osteosarcoma with ifosfamide: comparison of response in pediatric patients with recurrent disease versus patients previously untreated a Pediatric Oncology Group study. Med Pediatr Oncol 1995; 24: 87-92.

7 Rodríguez-Galindo C, Daw NC, Kaste SC, et al. Treatment of refractory osteosarcoma with fractionated cyclophosphamide and etoposide. J Pediatr Hematol Oncol 2002; 24: 250-55.

8 Berger M, Grignani G, Ferrari S, et al. Phase 2 trial of two courses of cyclophosphamide and etoposide for relapsed high-risk osteosarcoma patients. Cancer 2009; 115: 2980-87.

9 Navid F, Willert JR, McCarville MB, et al. Combination of gemcitabine and docetaxel in the treatment of children and young adults with refractory bone sarcoma. Cancer 2008; 113: 419-25.

10 Song BS, Seo J, Kim DH, Lim JS, Yoo JY, Lee JA. Gemcitabine and docetaxel for the treatment of children and adolescents with recurrent or refractory osteosarcoma: Korea Cancer Center Hospital experience. Pediatr Blood Cancer 2014; 61: 1376-81.

11 Duffaud F, Egerer G, Ferrari S, Rassam H, Boecker U, Bui-Nguyen B. A phase II trial of second-line pemetrexed in adults with advanced/ metastatic osteosarcoma. Eur J Cancer 2012; 48: 564-70.

12 Boye K, Del Prever AB, Eriksson M, et al. High-dose chemotherapy with stem cell rescue in the primary treatment of metastatic and pelvic osteosarcoma: final results of the ISG/SSG II study. Pediatr Blood Cancer 2014; 61: 840-45.

13 Sampson VB, Gorlick R, Kamara D, Anders Kolb E. A review of targeted therapies evaluated by the pediatric preclinical testing program for osteosarcoma. Front Oncol 2013; 3: 132.
14 Schwartz GK, Tap WD, Qin LX, et al. Cixutumumab and temsirolimus for patients with bone and soft-tissue sarcoma: a multicentre, open-label, phase 2 trial. Lancet Oncol 2013; 14: 371-82.

15 Pignochino Y, Grignani G, Cavalloni G, et al. Sorafenib block tumour growth, angiogenesis and metastatic potential in preclinica models of osteosarcoma through a mechanism potentially involving the inhibition of ERK1/2, MCL-1 and ezrin pathways. Mol Cancer 2009; 8: 118-30

16 Yang R, Piperdi S, Gorlick R. Activation of the RAF/mitogenactivated protein/extracellular signal-regulated kinase kinase/ extracellular signal-regulated kinase pathway mediates apoptosis induced by chelerythrine in osteosarcoma. Clin Cancer Res 2008; 14: 6396-404.

17 Kloos RT, Ringel MD, Knopp MV, et al. Phase II trial of sorafenib in metastatic thyroid cancer. J Clin Oncol 2009; 27: 1675-84.

18 Grignani G, Palmerini E, Dileo P, et al. A phase II trial of sorafenib in relapsed and unresectable high-grade osteosarcoma after failure of standard multimodal therapy: an Italian Sarcoma Group study. Ann Oncol 2012; 23: 508-16.

19 Pignochino Y, Dell'Aglio C, Basiricò M, et al. The Combination of Sorafenib and Everolimus Abrogates mTORC1 and mTORC2 upregulation in osteosarcoma preclinical models. Clin Cancer Res 2013; 19: 2117-31.

20 Amato RJ, Flaherty AL, Stepankiw M. Phase I trial of everolimus plus sorafenib for patients with advanced renal cell cancer. Clin Genitourin Cancer 2012; 10: 26-31.

21 Gomez-Martin C, Bustamante J, Castroagudin JF, et al. Efficacy and safety of sorafenib in combination with mammalian target of rapamycin inhibitors for recurrent hepatocellular carcinoma after liver transplantation. Liver Transpl 2012; 18: 45-52.

22 Harzstark AL, Small EJ, Weinberg VK, et al. A phase 1 study of everolimus and sorafenib for metastatic clear cell renal cell carcinoma. Cancer 2011; 117: 4194-200.

23 Eisenhauer EA, Therasse P, Bogaerts J, et al. New response evaluation criteria in solid tumours: revised RECIST guideline (version 1.1). Eur J Cancer 2009; 45: 228-47.

24 Tong D, Gillick L, Hendrickson FR. The palliation of symptomatic osseous metastases: final results of the Study by the Radiation Therapy Oncology Group. Cancer 1982; 50: 893-99.

25 Available at the URL address (last access date July 31, 2014) http:// www.npcrc.org/files/news/briefpain_short.pdf.

26 Simon R. Optimal two-stage designs for phase II clinical trials. Control Clin Trials 1989; 10: 1-10.

27 Bagatell R, Norris R, Ingle AM, et al. Phase 1 trial of temsirolimus in combination with irinotecan and temozolomide in children, adolescents and young adults with relapsed or refractory solid tumors: a Children's Oncology Group Study. Pediatr Blood Cancer 2014; 61: 833-39.

28 Luetke A, Meyers PA, Lewis I, Juergens H. Osteosarcoma treatmen - where do we stand? A state of the art review. Cancer Treat Rev 2014; 40: 523-32.

29 Leary SE, Wozniak AW, Billups CA, et al. Survival of pediatric patients after relapsed osteosarcoma: the St. Jude Children's Research Hospital experience. Cancer 2013; 119: 2645-53.

30 Van Glabbeke M, Verweij J, Judson I, Nielsen OS, and the EORTC Soft Tissue and Bone Sarcoma Group. Progression-free rate as the principal end-point for phase II trials in soft-tissue sarcomas. Eur J Cancer 2002; 38: 543-49.

31 Ray-Coquard I, Gelderblom H, Chevreau C, et al. An open-labe international multicentric phase II study of nilotinib in progressive pigmented villo-nodular synovitis (PVNS) not amenable to a conservative surgical treatment. Proc Am Soc Clin Oncol 2012; 30: abstr 10006.

32 Collins M, Wilhelm M, Conyers R, et al. Benefits and adverse events in younger versus older patients receiving neoadjuvant chemotherapy for osteosarcoma: findings from a meta-analysis. J Clin Oncol 2013; 31: 2303-12.

33 Baselga J, Campone M, Piccart M, et al. Everolimus in postmenopausal hormone-receptor-positive advanced breast cancer N Engl J Med 2012; 366: 520-29.

34 Escudier B, Eisen T, Stadler WM, et al, and the TARGET Study Group. Sorafenib in advanced clear-cell renal-cell carcinoma. N Engl J Med 2007; 356: 125-34. 
35 Motzer RJ, Escudier B, Oudard S, et al, and the RECORD-1 Study Group. Efficacy of everolimus in advanced renal cell carcinoma: a double-blind, randomised, placebo-controlled phase III trial. Lancet 2008; 372: 449-56.

36 Yao JC, Shah MH, Ito T, et al, and the RAD001 in Advanced Neuroendocrine Tumors, Third Trial (RADIANT-3) Study Group. Everolimus for advanced pancreatic neuroendocrine tumors. N Engl J Med 2011; 364: 514-23.

37 Le Cesne A, Van Glabbeke M, Verweij J, et al. Absence of progression as assessed by response evaluation criteria in solid tumors predicts survival in advanced GI stromal tumors treated with imatinib mesylate: the intergroup EORTC-ISG-AGITG phase III trial. J Clin Oncol 2009; 27: 3969-74.

38 Lencioni R, Llovet JM. Modified RECIST (mRECIST) assessment for hepatocellular carcinoma. Semin Liver Dis 2010; 30: 52-60.

39 Choi H, Charnsangavej C, Faria SC, et al. Correlation of computed tomography and positron emission tomography in patients with metastatic gastrointestinal stromal tumor treated at a single institution with imatinib mesylate: proposal of new computed tomography response criteria. J Clin Oncol 2007; 25: 1753-59.
40 Wilhelm SM, Carter C, Tang L, et al. BAY 43-9006 exhibits broad spectrum oral antitumor activity and targets the RAF/MEK/ERK pathway and receptor tyrosine kinases involved in tumor progression and angiogenesis. Cancer Res 2004; 64: 7099-109.

41 Fresno Vara JA, Casado E, de Castro J, Cejas P, Belda-Iniesta C, González-Barón M. PI3K/Akt signalling pathway and cancer. Cancer Treat Rev 2004; 30: 193-204.

42 Chen D, Zhao P, Li SQ, et al. Prognostic impact of pERK in advanced hepatocellular carcinoma patients treated with sorafenib. Eur J Surg Oncol 2013; 39: 974-80.

43 Adair JG. The Hawthorne Effect: a reconsideration of the methodological artifact. J Appl Psychol 1984; 69: 334-45. 